Free Radic Biol Med. 2015 March ; 80: 145-147. doi:10.1016/j.freeradbiomed.2015.02.005.

\title{
Introduction: What we do and do not know regarding redox processes of thiols in signaling pathways
}

\author{
Leslie B. Poole and \\ Department of Biochemistry, Wake Forest School of Medicine, Winston-Salem, North Carolina \\ 27157 USA

\section{Christian Schöneich} \\ Department of Pharmaceutical Chemistry, The University of Kansas, 2095 Constant Avenue, \\ Lawrence, KS 66047, USA
}

\begin{abstract}
Due to their susceptibility towards redox modification, protein thiols represent primary targets for the modulation of protein activity, conformation and oligomerization. Until fairly recently, such modifications were considered "damage" as a result of oxidative stress, before researchers recognized their physiological importance for biologic signaling. This paradigm shift, and the associated necessity to accurately characterize and quantify the various pathways of thiol redox modifications not only for specific proteins, but also within the cellular environment, has enticed researchers to take a close look at the impact of environment and molecular (protein) structure on these reactions. This Special Issue on Redox Biology of Thiols in Signaling Pathways is the result of a workshop organized at the 2013 Annual Meeting of the Society for Free Radical Biology and Medicine in San Antonio, Texas, summarizing the contributions from many of the presenters. It will provide a stimulating synopsis on what is known, and what is not known, about the reaction mechanisms which underlie the role of thiols and oxidative processes in signaling pathways.
\end{abstract}

\begin{abstract}
As we approach the 20-year mark since publication of the first, paradigm-shifting studies to link hydrogen peroxide production to growth factor signaling [1,2], it is sobering to realize that only a modest level of understanding has been gained of the molecular details behind how peroxide signals act to "shape" signaling pathways. Rather than simplifying the picture, the newly-recognized role for oxidants as positive effectors of cell signaling pathways challenged the view of oxidant defense systems as biological shields always at the ready to intercept and protect against the threat of harmful oxidants. And in this more complex regime, the abundant thiol groups in cells, including low molecular weight thiols and the thiol groups of cysteine-containing proteins, act in some cases as (i) redox buffers or active removal systems for minimizing oxidant damage, or in others as (ii) sensitive switches modulating protein function to achieve specific biological outcomes. Moreover, the general view that oxidant signaling is overwhelmingly the result of two-electron processes should not be so widely adopted that one electron processes become ignored as implausible, as the data available do not justify overlooking the potential for radical-based signaling pathways
\end{abstract}


to be important factors in cell signaling. In the review articles that follow, provocative theories or models of free radical reactions involved in cellular signaling are discussed $[3,4]$. In addition, two of the reviews present the intertwined enzymology and cell biology of oxidative protein folding [5] and peroxide defense [6]. As technological advances in evaluating the role of biological thiols in cell signaling have been critical for the insights that we do now appreciate since "redox regulation and signaling" became a recognized phenomenon, we include a comprehensive review on methods and approaches to assess thiol oxidation that have been at the heart of many of these advances [7].

\section{Understanding the basics}

This issue leads with a review covering of some of the relevant biochemical and biophysical properties of cysteines and small molecule thiols at a level that is intended for educating nonspecialists in thiol-linked chemistry [8]. This is particularly important as the relevance of redox biology and thiol modifications reaches farther and farther into many biological contexts, providing a challenge to investigators lacking an adequate background in this subject. Bioinformatic studies indicate that cysteine, as one of the least abundant amino acids, is found at high frequency in functional sites given its unique chemical attributes, including its tendency to ionize (losing a proton to generate the thiolate form, $\mathrm{R}_{-} \mathrm{S}^{-}$) and act as a nucleophile in $\mathrm{S}_{\mathrm{N}} 2$ displacement reactions [8]. Cysteines are also sites of adduct formation with cellular electrophiles and xenobiotics, and such reactivities can underlie effective cell signaling processes or irreversible oxidative damage, depending on the nature and location of the modification in responsive proteins [9]. Metal binding sites, including biologically-important $\mathrm{Zn}$ fingers and iron sulfur clusters, are also sites that rely on such functional cysteine residues [10]. The context of cysteine residues within the microenvironment of folded proteins exerts the dominant influence on the reactivity of specific cysteine residues, as attributes such as $\mathrm{pK}_{\mathrm{a}}$, redox potential and the constellation of residues surrounding the thiol group set the stage for the regulatory, catalytic or binding functions exhibited by these sites [8]. While some of these features have long been appreciated by biochemists, we still have much to learn about the detailed biochemistry and actual biological roles that cysteine chemistry plays in living systems.

\section{One electron chemistry and signaling}

Two articles follow which examine the one-electron redox chemistry of thiols and its potential contribution to signaling. The one-electron oxidation of thiols produces thiyl radicals $\left(\mathrm{RS}^{\bullet}\right)$, which can rapidly react with molecular oxygen, nitric oxide (NO) and various other radical and non-radical molecules of biologic significance. The reaction of thiyl radicals with NO is discussed as one potential mechanism for the formation of Snitrosothiols [11], which represent important mediators of thiol-dependent signaling. Thiyl radicals are also key intermediates in nucleotide exchange reactions of various GTPases [12]. Recent work, though, has demonstrated that protein thiyl radicals may equilibrate with carbon-centered radicals through various intramolecular hydrogen atom transfer (HAT) mechanisms. These carbon-centered radicals can convert into peroxyl radicals through the reaction with molecular oxygen. When carbon-centered radicals are generated at the acarbon of the polypeptide backbone, reverse HAT may lead to epimerization, i.e. the 
generation of D-amino acids, if permitted by protein conformation and dynamics. Based on rate constants for these HAT reactions, Nauser et al. [3] provide kinetic simulations which show that products derived from intramolecular HAT reactions can make up a significant fraction of the final reaction products generated from protein thiyl radicals. The yields of these products increase when concentrations of antioxidants decrease, for example as a result of oxidative stress. Depending on the environment, the intramolecular HAT pathways are expected to compete with thiyl radical reactions underlying signaling processes, i.e. the reaction of thiyl radicals with $\mathrm{NO}$ or glutathione (thiolate) and molecular oxygen.

Unresolved questions also remain with regard to the biologic generation of thiyl radicals for signaling processes [4]. Without doubt radical reactions with thiols would be sufficiently fast to enable a rapid cellular response to stimuli. However, the lack of selectivity of these reactions raises concerns. A potential way to gauge reactions of radicals towards specific protein thiols would be the thiol $\mathrm{pK}_{\mathrm{a}}$, where specific radicals react more efficiently with protonated thiol and others with thiolate. However, the removal of radicals by cellular antioxidants, including glutathione, would question the efficiency by which thiyl radicals may be generated for signaling. The lack of selectivity of radicals is in stark contrast to reactions of hydrogen peroxide with thiols, which, in most cases, are probably too slow. Arguments against a general role of hydrogen peroxide-mediated oxidation of thiols during signaling also rely on the fact that cells encounter a low steady-state concentration of hydrogen peroxide. This raises the question on how a cell would differentiate between steady-state and signal-triggering levels of hydrogen peroxide.

\section{Oxidative protein folding}

In order for many secreted or endoplasmic reticulum (ER)-resident proteins to fold properly in eukaryotic systems, disulfide bonds must be inserted and isomerized through processes that are still incompletely understood. The net generation of disulfide bonds from dithiols is a two-electron oxidation process that can be catalyzed in the ER by several enzymatic systems that oxidize members of the protein disulfide isomerase (PDI) family, including Ero1, the peroxiredoxin Prdx4, glutathione peroxidase (Gpx7/8), and Vitamin K epoxide oxidoreductase (VKOR) [5]. In these so-called "PDI first" pathways, the oxidized PDI then participates in thiol-disulfide exchange reactions to install disulfide bonds in client proteins. An alternative pathway has also been identified, catalyzed by the quiescin sulfhydryl oxidase (QSOX) family of enzymes, that does not require PDI for the initial disulfide bond formation, but rather oxidizes unfolded peptides and proteins directly through the transfer of electrons to molecular oxygen [13]. In this "PDI second" scenario, the role of the PDI is solely as an isomerase. This is because, however the disulfide bonds are initially introduced into the unfolded protein or peptide substrates, this process is inherently error prone and thiol-disulfide exchange catalyzed by PDIs is required to generate the correct native disulfide bonds in the properly folded protein products. Thus, while PDI is inevitably involved with oxidative protein folding in the ER, models differ in whether or not PDI is needed for the net generation of disulfide bonds in ER proteins. There are also considerable questions remaining as to the role the prevalent thiol-containing small molecule glutathione plays in oxidative protein folding. As highlighted in the contribution by Thorpe and colleagues in this series [5], emerging approaches enabling trapping and analysis of redox 
states of multiple proteins simultaneously offer great promise that the relative contributions of the various redox components in the ER can ultimately be sorted out through systemslevel analyses and mutational effects.

\section{Peroxiredoxin structure and function}

While catalase and glutathione peroxidases have long been recognized as important peroxide defense systems, peroxiredoxins (Prxs) have been recognized only relatively recently as powerful peroxide removal systems that are even more widespread in biology. Lacking the bound heme or selenocysteine typically found in catalase or glutathione peroxidases, respectively, the Prxs rely on a single cysteine thiol within a specialized active site that can react with peroxide substrates with rates in excess of $10^{7} \mathrm{M}^{-1} \mathrm{~s}^{-1}$, putting them on a par (in reactivity) with the more widely recognized defense enzymes [6]. Moreover, nearly all organisms and cell types express at least one of these Prx isoforms at high levels, supporting the view that these proteins are perhaps the most important peroxide (and peroxynitrite) defense enzymes in biology.

For many Prxs, recycling of the active site cysteine that becomes oxidized during catalysis occurs through participation of a second cysteine residue commonly coming from the Prx itself (referred to as the resolving cysteine), but the efficiency with which subsequent disulfide bond formation takes place varies greatly among Prxs and provides an opportunity for this cysteine to act as a point of regulation in these enzymes. Upon reaction with a peroxide, the cysteine is converted to sulfenic acid (R-SOH); the sulfenic acid then typically reacts with the resolving cysteine to yield a disulfide, undergoing subsequent reduction to allow for another catalytic cycle. However, when peroxide levels are high and disulfide bond formation is slow, the sulfenic acid can be further oxidized to form a sulfinic acid (R$\mathrm{SO}_{2} \mathrm{H}$ ). Most Prxs susceptible to this oxidative inactivation are eukaryotic, and the presence of a specialized enzyme, sulfiredoxin, to repair this oxidatively inactive form of Prxs argues that this process is biologically relevant. Putative roles for this evolutionarily-selected oxidative inactivation sensitivity include aspects of both the loss of peroxidase function (e.g., to allow for $\mathrm{H}_{2} \mathrm{O}_{2}$ to build up and serve as a signaling molecule and/or to conserve reducing power for other cellular needs under oxidative stress) or gain of additional functions (i.e., as a molecular chaperone or as a "fire alarm" signal), summarized in the contribution by Karplus and elsewhere [6,14].

\section{Technologies and approaches for exploring thiol biology and chemistry}

Analytical capabilities for determining cysteine amounts and modifications have expanded dramatically in recent years with the enhancement in mass spectrometry (MS) approaches to analyze biomolecules, opening the door to a better understanding of protein thiol chemistry, biochemistry and biology. In particular, since the introduction of "soft ionization" techniques like electrospray ionization and soft laser desorption (for which the Nobel Prize was awarded to Fenn and Tanaka, respectively, in 2002), the application of mass spectrometry to analyze biological samples has greatly expanded. Proteins and peptides are routinely sequenced and identified, including those associated in non-covalent complexes, post-synthesis modifications on proteins and nucleic acids are increasingly amenable to MS 
detection and analysis, and a wide range of metabolites can be identified, all by combining powerful labeling approaches and chemical tools with high performance MS instrumentation. In the final article of this series, Furdui and colleagues begin with a background for the non-practitioner on the principles of MS and basic components of MS instrumentation [7]. They then describe a number of applications of MS to investigate the chemistry and kinetics underlying protein thiol oxoform formation, reactivity, and repair using intact thiol-containing proteins as model systems. The development of chemical agents and tailored workflow strategies have been important for analyzing the thiols or thiolderived modifications of proteins and small molecules in complex samples, as well, and experimental strategies to identify protein oxoforms in proteins and peptides are described in the final major section of this review [7]. The authors conclude with remarks about the challenges that remain and their vision for the future, including the integration of oxidative post-translational modification information into computational models of cellular networks, as we seek to gain clarity as to the nature of the contributions that thiol groups make in thiolbased signaling and regulation.

\section{Acknowledgments}

The authors are grateful for support from the National Institutes of Health (R01 GM050389 and R33 CA177461 to L.B.P., and P01AG12993 to C.S).

\section{References}

1. Chen Q, Olashaw N, Wu J. Participation of reactive oxygen species in the lysophosphatidic acidstimulated mitogen-activated protein kinase kinase activation pathway. J Biol Chem. 1995; 270:28499-28502. [PubMed: 7499358]

2. Sundaresan M, Yu ZX, Ferrans VJ, Irani K, Finkel T. Requirement for generation of $\mathrm{H} 2 \mathrm{O} 2$ for platelet-derived growth factor signal transduction. Science. 1995; 270:296-299. [PubMed: 7569979]

3. Nauser T, Koppenol WH, Schoneich C. Protein thiyl radical reactions and product formation: a kinetic simulation. Free Radic Biol Med. 2015; 80:158-163. [PubMed: 25499854]

4. Winterbourn CC. Are free radicals involved in thiol-based redox signaling? Free Radic Biol Med. 2015; 80:164-170. [PubMed: 25277419]

5. Hudson DA, Gannon SA, Thorpe C. Oxidative protein folding: From thiol-disulfide exchange reactions to the redox poise of the endoplasmic reticulum. Free Radic Biol Med. 2015; 80:171-182. [PubMed: 25091901]

6. Karplus PA. A primer on peroxiredoxin biochemistry. Free Radic Biol Med. 2015; 80:183-190. [PubMed: 25452140]

7. Devarie Baez NO, Reisz JA, Furdui CM. Mass Spectrometry in Studies of Protein Thiol Chemistry and Signaling: Opportunities and Caveats. Free Radic Biol Med. 2015; 80:191-211. [PubMed: 25261734]

8. Poole LB. The basics of thiols and cysteines in redox biology and chemistry. Free Radic Biol Med. 2015; 80:148-157. [PubMed: 25433365]

9. Higdon A, Diers AR, Oh JY, Landar A, Darley-Usmar VM. Cell signalling by reactive lipid species: new concepts and molecular mechanisms. Biochem J. 2012; 442:453-464. [PubMed: 22364280]

10. Pace NJ, Weerapana E. Diverse functional roles of reactive cysteines. ACS chemical biology. 2013; 8:283-296. [PubMed: 23163700]

11. Jourd'heuil D, Jourd'heuil FL, Feelisch M. Oxidation and nitrosation of thiols at low micromolar exposure to nitric oxide. Evidence for a free radical mechanism. J Biol Chem. 2003; 278:15720 15726. [PubMed: 12595536] 
12. Mitchell L, Hobbs GA, Aghajanian A, Campbell SL. Redox regulation of Ras and Rho GTPases: mechanism and function. Antioxid Redox Signal. 2013; 18:250-258. [PubMed: 22657737]

13. Kodali VK, Thorpe C. Oxidative protein folding and the Quiescin-sulfhydryl oxidase family of flavoproteins. Antioxid Redox Signal. 2010; 13:1217-1230. [PubMed: 20136510]

14. Karplus PA, Poole LB. Peroxiredoxins as molecular triage agents, sacrificing themselves to enhance cell survival during a peroxide attack. Mol Cell. 2012; 45:275-278. [PubMed: 22325349] 